\title{
Surface roughness and interfacial slip boundary condition for quartz crystal microbalances
}

\author{
G. McHale ${ }^{\text {a) }}$ and M. I. Newton \\ School of Science, The Nottingham Trent University, Clifton Lane, Nottingham NG11 8NS, United Kingdom
}

(Received 15 April 2003; accepted 8 October 2003)

\begin{abstract}
The response of a quartz crystal microbalance (QCM) is considered using a wave equation for the substrate and the Navier-Stokes equations for a finite liquid layer under a slip boundary condition. It is shown that when the slip length to shear wave penetration depth is small, the first-order effect of slip is only present in the frequency response. Importantly, in this approximation the frequency response satisfies an additivity relation with a net response equal to a Kanazawa liquid term plus an additional Sauerbrey "rigid" liquid mass. For the slip length to result in an enhanced frequency decrease compared to a no-slip boundary condition, it is shown that the slip length must be negative so that the slip plane is located on the liquid side of the interface. It is argued that the physical application of such a negative slip length could be to the liquid phase response of a QCM with a completely wetted rough surface. Effectively, the model recovers the starting assumption of additivity used in the trapped mass model for the liquid phase response of a QCM having a rough surface. When applying the slip boundary condition to the rough surface problem, slip is not at a molecular level, but is a formal hydrodynamic boundary condition which relates the response of the QCM to that expected from a QCM with a smooth surface. Finally, possible interpretations of the results in terms of acoustic reflectivity are developed and the potential limitations of the additivity result should vapor trapping occur are discussed. (C) 2004 American Institute of Physics.
\end{abstract}

[DOI: 10.1063/1.1630373]

\section{INTRODUCTION}

A quartz crystal microbalance (QCM) responds to immersion in a liquid via changes in its resonant frequency and damping. These energy storage and energy dissipation effects are sensitive probes of the interface between the crystal and liquid. The interfacial region is defined by the viscous entrainment of liquid within a penetration depth $\delta$ $=\left(2 \eta_{f} / \omega \rho_{f}\right)^{1 / 2}$ where $\eta_{f}$ is the viscosity, $\rho_{f}$ is the density, and $\omega=2 \pi f$ is the angular frequency. It has long been known that a crystal with a rough surface has an excess liquid phase response, primarily in its frequency decrease, compared to that predicted by the Kanazawa-Gordon equation. ${ }^{1-13}$ One suggested method of accounting for this response has been to view the response as composed of a Kanazawa term ${ }^{14}$ accounting for the entrainment of the liquid plus a Sauerbrey ${ }^{15}$ rigid-mass-type term with the mass being given by the liquid "trapped" within the surface structure of the crystal. ${ }^{1,8,13}$ The assumed additivity of these terms has been the starting point of the model and has not been directly derived from any wave equation for the system. It has also been shown experimentally that the state of hydrophobicity/hydrophilicity of the surface of a QCM can influence its response ${ }^{5,9,11}$ even when the surface is relatively smooth $^{9}$ (i.e., surface features of depth $<0.05 \mu \mathrm{m}$ ). The discussion of excess response due to roughness has, at times inevitably, become entangled with the state of wetting of a surface and possible interfacial slip at the molecular

\footnotetext{
a) Author to whom correspondence should be addressed. Electronic mail: glen.mchale@ntu.ac.uk
}

level. ${ }^{9-11}$ This present report does not argue for or against either a dominantly roughness-induced response or a partially molecular-slip-induced response. However, we believe that whether molecular slip can occur in the liquid phase response of a QCM and whether its effects can be separated from a roughness-induced response is a valid issue, particularly when dealing with surfaces chemically modified for biosensing experiments. It is therefore extremely important to recognize that a slip boundary condition is a precise mathematical condition, which can lead to specific predictions that can be tested against any anomalous response observed in an experiment.

The concept of interfacial slip is precisely defined in terms of a slip boundary condition, which gives a discontinuity between the solid and liquid velocities at the interface, ${ }^{16-18}$ earlier attempts at devising models to describe possible molecular slip occurring in QCMs included a complex slip parameter ${ }^{19}$ and an interfacial layer model. ${ }^{20}$ To create a mathematical relation for a slip boundary condition does not assign a physical origin to the slip parameter. In one sense a slip parameter may be a mechanism to account for a diffuse interface, while in another it may relate directly to a discontinuity of the first molecular layer of the liquid. In this article, we consider the relationship between load impedance derived with and without a slip boundary condition. We neither prove nor disprove the existence of molecular slip in the liquid phase QCM response. A key focus of the article is to address the application of the slip boundary condition to model the response when a crystal with a rough surface is immersed in a Newtonian liquid; the use of a slip boundary condition in this situation does not necessarily imply slip is 


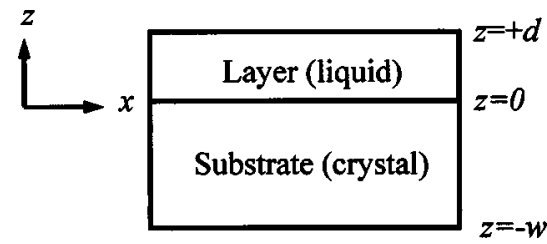

FIG. 1. Definition of axes for quartz crystal substrate with a liquid overlayer.

occurring in the first molecular layer of liquid. We show rigorously that under the condition that a rough surface is completely wetted by a liquid, a slip boundary condition can, under appropriate conditions on the size of roughness, result in the additivity of a Kanazawa-Gordon term with a Sauerbrey trapped liquid mass term for the frequency response; to first order the motional resistance, representing dissipation, is independent of the roughness. The model is developed in terms of a liquid layer of finite thickness rather than simply an infinitely deep Newtonian liquid. The mathematical development of this model is given in a fully self-contained manner in Sec. II with the necessary experimental consequences of the model in Secs. III A and III B. Possible limitations in the one-dimensional nature of the model of the substrate and in extending the additivity to the case of trapped air/vapor in surface features are discussed in the context of acoustic reflectivity of the solid-liquid and solid-air interface in Secs. III C and III D. The situation of partial penetration of liquid into surface features is relevant for hydrophobic or partially wetting QCM surfaces.

\section{THEORY}

\section{A. Wave equations}

A first-principles model of the response of a smooth QCM to loading by a finite liquid layer (Fig. 1) can be obtained by setting up an essentially one-dimensional wave equation for the substrate of thickness $w$ and the NavierStokes equations for the liquid layer of thickness $d$. The equations can be solved and boundary conditions applied at the various interfaces to obtain the displacements or speed of motion of both the substrate and layer. Several routes are then possible to obtain the effect of the layer on the resonant frequency and damping of the substrate. In the first case, a load impedance method, which relates the shear stress to the substrate speed at the interface, can be used..$^{21}$ Alternatively a perturbation expansion can be adopted about the resonant frequency of the unloaded substrate. ${ }^{22}$ Either method is possible, and both should provide the same results, although many experimental studies use the formalism of the load impedance method.

The Navier-Stokes equation for a Newtonian liquid, and assuming continuity and incompressibility, has an equation for fluid flow,

$$
\frac{\eta_{f}}{\rho_{f}} \nabla^{2} \underline{\underline{v}}_{f}=i \omega \underline{v}_{f}
$$

where $\rho_{f}$ is the density of the fluid, $\eta_{f}$ is the viscosity of the fluid, $\underline{v}_{f}$ is the fluid velocity, $\omega$ is the angular frequency, and a time dependence $e^{i \omega t}$ has been assumed. The substrate displacement $\underline{u}_{s}$ must satisfy the wave equation

$$
\nabla^{2} \underline{u}_{s}=-\frac{\omega^{2}}{c_{s}^{2}} \underline{u}_{s},
$$

where $c_{s}=\left(\mu_{s} / \rho_{s}\right)^{1 / 2}$ is the intrinsic shear speed of the substrate material determined by its shear modulus $\mu_{s}$ and density $\rho_{s}$. Solutions to these equations of motion can be sought using velocity and displacement functions of the form

$$
\underline{v}_{f}=\left(v_{f}(z) e^{i \omega t}, 0,0\right)
$$

and

$$
\underline{u}_{s}=\left(u_{s}(z) e^{i \omega t}, 0,0\right) .
$$

Because the substrate is smooth, the displacement, Eq. (4), is essentially one dimensional. Substituting Eqs. (3) and (4) into Eqs. (1) and (2) and recognizing that the general solutions are composed of exponentials gives the general solutions

$$
v_{f}(z)=A_{f} \exp \left(i k_{f} z\right)+B_{f} \exp \left(-i k_{f} z\right)
$$

and

$$
u_{s}(z)=A_{s} \exp \left(i k_{s} z\right)+B_{s} \exp \left(-i k_{s} z\right),
$$

where the $k$ vectors are given by $k_{f}=(-2 i)^{1 / 2} / \delta$ and $k_{s}$ $=\omega / c_{s}$, the $A_{i}$ and $B_{i}$ are constants determined by boundary conditions, and the fluid wave vector has been written using the shear-wave penetration depth $\delta=\left(2 \eta_{f} / \omega \rho_{f}\right)^{1 / 2}$.

To convert from a general solution to a specific solution boundary conditions must be imposed at the upper and lower free surfaces and at the interface between the substrate and layer. Only the latter of these conditions depends upon the slip or no-slip boundary condition and we, therefore, first develop the form of the solution using the boundary condition of vanishing shear stress at the upper and lower free surfaces of the substrate-fluid layer system, i.e.,

$$
\eta_{f}\left(\frac{\partial v_{f}}{\partial z}\right)_{z=d}=0
$$

and

$$
\mu_{s}\left(\frac{\partial u_{s}}{\partial z}\right)_{z=-w}=0 .
$$

Using Eqs. (4) and (5) in Eqs. (7) and (8) determines two of the four constants $A_{f}, B_{f}, A_{s}$, and $B_{s}$, so that the solutions become

$$
v_{f}(z)=2 A_{f} \exp \left(\frac{\sqrt{2 i} d}{\delta}\right) \cosh \left[\frac{\sqrt{2 i}(z-d)}{\delta}\right]
$$

and

$$
u_{s}(z)=2 A_{s} \exp \left(-i k_{s} w\right) \cos \left[k_{s}(z+w)\right] .
$$

The relationship between the two remaining constants $A_{f}$ and $A_{s}$ is determined by the boundary condition still to be imposed at the substrate-fluid layer interface. It is interesting to note that due to the complex argument in the $\cosh (\cdots)$, 
whether or not a slip boundary condition is chosen, the fluid velocity will have a damped oscillation representing viscous entrainment with a penetration into the (fluid) layer set by the shear-wave penetration depth $\delta$. The derivations in this section can be extended to the case of a substrate coated by a viscoelastic layer; both the cases of slip of a liquid or a solid layer on a QCM surface can then be obtained by taking the appropriate limits. For completeness, the key equations for a derivation for the viscoelastic case are given in Appendix A.

\section{B. Surface mechanical impedance}

To obtain the usual Kanazawa-Gordon and Sauerbrey equations we could now develop a perturbation expansion about a vanishing thickness liquid layer. The alternative we adopt here is to use the surface mechanical impedance of the film, ${ }^{17,23} Z_{L}$, defined by

$$
Z_{L}=\left[\frac{F_{f}}{v_{s}}\right]_{z=0},
$$

where $F_{f}$ is the shear force exerted by the film on the substrate per unit area and is given by the shear stress:

$$
F_{f}=-\eta_{f}\left(\frac{d v_{f}}{d z}\right)_{z=0} .
$$

In a linear approximation the relationships between the load impedance and angular frequency shift and dissipation are given by

$$
\Delta \omega=\frac{-1}{\rho_{s} w} \operatorname{Im}\left[Z_{L}\right]
$$

and

$$
\Delta D=\frac{2}{\omega \rho_{s} w} \operatorname{Re}\left[Z_{L}\right]
$$

The dissipation $\Delta D$ can be related directly to the motional resistance $R_{m}$, and the substrate thickness determines the resonant frequency via $w=m \pi c_{s} / \omega$ with $m=1$ giving the fundamental frequency of the QCM. Using Eqs. (9)-(12) an expression can be developed for the impedance in the following form that is not specific to whether a slip or no-slip boundary condition is to be applied:

$$
\begin{aligned}
Z_{L}= & -i \sqrt{\frac{i \rho_{f} \eta_{f}}{\omega}}\left(\frac{A_{f}}{A_{s}}\right) \exp \left(i k_{s} w+\frac{\sqrt{2 i} d}{\delta}\right) \\
& \times\left[\frac{\sinh \left(\frac{\sqrt{2 i} d}{\delta}\right)}{\cos \left(k_{s} w\right)}\right] .
\end{aligned}
$$

Thus, the surface load impedance is proportional to $A_{f} / A_{s}$ so that the sensitivity to the precise boundary condition at the substrate-fluid layer interface enters the impedance through the relationship between $A_{f}$ and $A_{s}$. Appendix A gives the analogous results for a finite viscoelastic layer.

\section{Substrate-layer interface boundary conditions}

\section{No-slip boundary condition}

The no-slip condition imposes the condition that fluid velocity and substrate velocity should be equal at the boundary between the substrate and layer; equivalently, the displacements can be matched. Using Eqs. (9) and (10) and setting $v_{f}(z=0)=i \omega u_{s}(z=0)$ gives

$$
\begin{aligned}
A_{f}^{n o ~ s l i p}= & i \omega \exp \left(-i k_{s} w+\frac{\sqrt{2 i} d}{\delta}\right) \\
& \times\left[\frac{\cos \left(k_{s} w\right)}{\cosh \left[\frac{\sqrt{2 i} d}{\delta}\right]}\right] A_{s}^{n o s l i p},
\end{aligned}
$$

where the superscript no slip has been introduced to remind us that the no-slip boundary condition has been used to determine the relationship between the constants $A_{f}$ and $A_{s}$. Using Eq. (15) we then obtain the impedance

$$
Z_{L}^{\text {no slip }}=\sqrt{i \omega \rho_{f} \eta_{f}} \tanh \left[\frac{\sqrt{2 i} d}{\delta}\right] .
$$

\section{Slip boundary condition}

In an earlier report we used a slip boundary condition introduced by Rodahl and Kasemo ${ }^{16}$ (see also McHale et $a l .{ }^{17}$ ) which related the mismatch in speeds at the boundary between the substrate and layer to the shear stress at the boundary, i.e.,

$$
\chi m_{M L}\left[v_{s}(z=0)-v_{f}(z=0)\right]=F_{f},
$$

where $\chi$ is the coefficient of friction between the film and surface and $m_{M L}$ is the mass per unit area of a monolayer of the film. In an earlier report ${ }^{17}$ we introduced an $s$ factor defined as $s=1 / \chi m_{M L}$. In contrast, Ellis and Hayward ${ }^{18}$ have recently introduced a slip length $b$ defined by the boundary condition

$$
v_{s}(z=0)=v_{f}(z=-b) .
$$

Performing a Taylor expansion of Eq. (19) about $z=0$ gives

$$
v_{s}(z=0)-v_{f}(z=0)=-b\left(\frac{d v_{f}}{d z}\right)_{z=0},
$$

and with the definition of $F_{f}$ used in Eq. (12) this gives

$$
\frac{\eta_{f}}{b}\left[v_{s}(z=0)-v_{f}(z=0)\right]=F_{f} .
$$

Comparing Eq. (21) to Eq. (18) we deduce that $s=b / \eta_{f}$. The relationship between the fluid layer velocity gradient extrapolated from the bulk and the slip length $b$ is shown diagrammatically in Fig. 2. The slip boundary condition, Eq. (18), can therefore be regarded as a first-order approximation to the slip boundary condition in Eq. (19) so that the two slip boundary conditions are consistent with each other. In the case of a viscoelastic rather than a liquid layer, the equivalent relation for $s$ is $s=i \omega b / G_{f}$, where $G_{f}$ is the complex shear modulus (see Appendix B). 


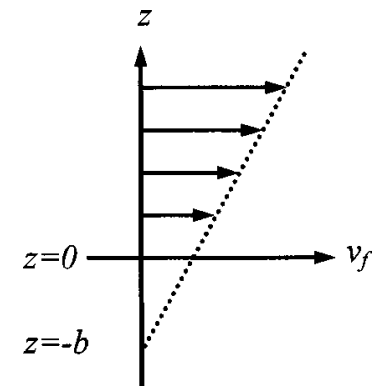

FIG. 2. Extrapolation of the fluid speed gradient from the bulk liquid and the relationship to the slip parameter $b$.

Applying the Ellis-Hayward ${ }^{18}$ slip boundary condition [Eq. (19)] to Eqs. (9) and (10) gives

$$
\begin{aligned}
A_{f}^{s l i p}= & i \omega \exp \left(-i k_{s} w-\frac{\sqrt{2 i} d}{\delta}\right) \\
& \times\left[\frac{\cos \left(k_{s} w\right)}{\cosh \left[\frac{\sqrt{2 i}(d+b)}{\delta}\right]}\right] A_{s}^{s l i p},
\end{aligned}
$$

which differs from the no-slip case only by a shift of $d$ by $b$ in the $\cosh (\cdots)$ term in the denominator of Eq. (22). Using the definition of the surface mechanical impedance we obtain

$$
Z_{L}^{s l i p}=\frac{\sqrt{i \omega \rho_{f} \eta_{f}} \sinh \left(\frac{\sqrt{2 i} d}{\delta}\right)}{\cosh \left(\frac{\sqrt{2 i}(d+b)}{\delta}\right)},
$$

which reduces to the no-slip result when $b=0$.

In the case that the dimensionless combination characterizing the influence of $\operatorname{slip} b / \delta$ is small, the $\cosh (\cdots)$ can be expanded as

$$
\begin{aligned}
\cosh \left(\frac{\sqrt{2 i}(d+b)}{\delta}\right) \approx & \cosh \left(\frac{\sqrt{2 i} d}{\delta}\right) \\
& \times\left[1+\frac{\sqrt{2 i} b}{\delta} \tanh \left(\frac{\sqrt{2 i} d}{\delta}\right)\right],
\end{aligned}
$$

and the impedance, Eq. (23), becomes

$$
Z_{L}^{\text {slip }} \approx \frac{\sqrt{i \omega \rho_{f} \eta_{f}} \tanh \left(\frac{\sqrt{2 i} d}{\delta}\right)}{1+\frac{\sqrt{2 i} b}{\delta} \tanh \left(\frac{\sqrt{2 i} d}{\delta}\right)} .
$$

Equation (25) can be rewritten in the form

$$
Z_{L}^{s l i p}=\frac{Z_{L}^{\text {no slip }}}{1+\frac{b}{\eta_{f}} Z_{L}^{\text {no slip }} .}
$$

The factor $b / \eta_{f}$ in the denominator of Eq. (26) is the slip factor $s$. We have previously derived Eq. (26) using a harmonic oscillator substrate model coated by a general finite viscoelastic layer and have shown that it can be interpreted using a single-loop feedback model; ${ }^{17}$ this equation can also be derived from the general interfacial layer approach of Ref. 17. Appendix B gives the analogous results for a viscoelastic layer and hence includes both the solid and liquid limits.

\section{DISCUSSION}

\section{A. "Liquid" mass layer additivity}

The idea of a "rigid" liquid mass added to a Kanazawatype entrained liquid response is implicit with Eq. (26). To show this rigorously we expand Eq. (26),

$$
Z_{L}^{\text {slip }} \approx Z_{L}^{\text {no } \operatorname{slip}}\left(1-\frac{b}{\eta_{f}} Z_{L}^{\text {no slip }}\right),
$$

and consider the layer to be an infinitely deep Newtonian fluid, so that Eq. (17) gives

$$
Z_{L}^{n o} \operatorname{slip} \approx \sqrt{i \omega \rho_{f} \eta_{f}} .
$$

Since $(2 i)^{1 / 2}=1+i$, Eq. (27) becomes

$$
Z_{L}^{s l i p} \approx(1+i) \sqrt{\frac{\omega \rho_{f} \eta_{f}}{2}}\left(1-\frac{(1+i) b}{\eta_{f}} \sqrt{\frac{\omega \rho_{f} \eta_{f}}{2}}\right),
$$

which after expanding, grouping terms into real and imaginary, and using the definition of the penetration depth gives

$$
Z_{L}^{s l i p} \approx \sqrt{\frac{\omega \rho_{f} \eta_{f}}{2}}\left[1+i\left(1-\frac{2 b}{\delta}\right)\right] .
$$

Another view of Eq. (30) is that the impedance for an infinitely deep Newtonian liquid using the slip boundary condition contains the Kanazawa result assuming a no-slip boundary condition plus an additional impedance equal to $-i \omega \rho_{f} b$; the analogous result for a thin layer of rigid mass is given Appendix C. The real part of the impedance, Eq. (30), gives the dissipation due to the liquid, and since it does not include a slip correction factor, it is relatively insensitive to the slip length in this approximation of small $b / \delta$. In contrast, the imaginary part of the impedance, which determines the frequency shift, has a correction factor involving the slip length parameter. Using Eq. (13) and the fundamental resonance condition $w=\pi v_{s} / \omega$, Eq. (30) gives

$$
\left(\frac{\Delta \omega}{\omega}\right)_{\text {slip }} \approx\left(\frac{\Delta \omega}{\omega}\right)_{\text {no slip }}\left(1-\frac{2 b}{\delta}\right),
$$

where

$$
\left(\frac{\Delta \omega}{\omega}\right)_{n o \text { slip }} \approx-\frac{1}{\pi} \sqrt{\frac{\omega \rho_{f} \eta_{f}}{2 \rho_{s} \mu_{s}}} .
$$

Combining Eq. (32) with the additional factor $2 b / \delta$ occurring in Eq. (31) gives

$$
\left(\frac{\Delta \omega}{\omega}\right)_{\text {additional }} \approx\left(-\frac{2 b}{\delta}\right)\left(\frac{\Delta \omega}{\omega}\right)_{\text {no slip }}=\frac{\omega \Delta m_{f}}{\pi \sqrt{\mu_{s} \rho_{s}}},
$$

where $\Delta m_{f}=b \rho_{f}$ has been defined. Equation (33) is of the Sauerbrey form for a frequency shift due to a rigid "liquid" mass per unit area deposited on a smooth substrate (quartz crystal); for the case of a thin mass layer given in Appendix $\mathrm{C}$ the additional term is not of a mass-type form and is expected to be a small correction to the Sauerbrey result. Equa- 


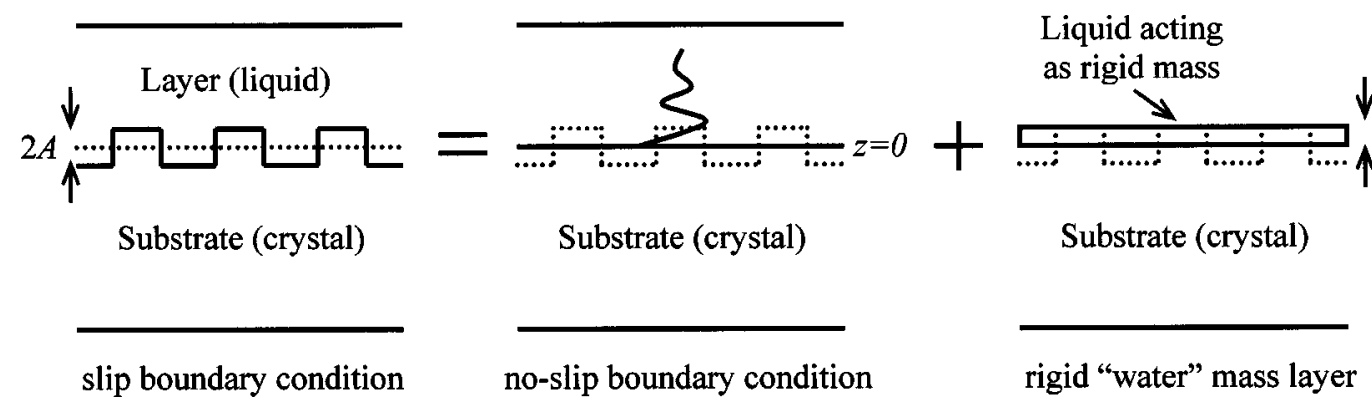

FIG. 3. Diagrammatic interpretation of Eqs. (31)-(33). The frequency response of a smooth QCM to immersion in water is treated by the slip boundary condition as a perfect liquid entrainment by a smooth crystal with a no-slip boundary condition plus an additional component equal to a layer of "rigid water" of thickness $|b|$. The dotted lines indicate a hypothetical "rough" surface whose average position lies at $z=0$.

tion (30) predicts a dominant first-order effect in the frequency shift rather than the dissipation, but any conversion of the shear motion in the liquid into nonshear motion by, for example, strong roughness or oblique angles in the surface roughness or topography is likely to generate compressional waves and hence significant damping of the QCM.

One difficulty with the additional mass interpretation of Eq. (33) would be that a positive value for $b$ would give a frequency increase, whereas added mass of the Sauerbrey form should give a frequency decrease. A positive value for the slip parameter $b$ places the slip plane into the solid side of the boundary while a negative value places the slip plane out into the liquid side of the boundary (see Fig. 2). Diagrammatically, Eqs. (31)-(33) mean that the frequency response of a smooth crystal (substrate) with a slip boundary condition and a negative slip parameter $b=-|b|$ can be viewed as the sum of the effect of liquid entrainment using a no-slip boundary condition plus a "rigid" mass layer of thickness $|b|$ and density $\rho_{f}$ (see Fig. 3). Given some of the confusion that exists in the literature on acoustic wave sensors and slip, it should be emphasized that the development of the equations so far in this work has no physical meaning beyond the mathematical condition of a discontinuity in the substrate and liquid velocities at the solid-liquid interface. Should such a discontinuity occur by some physical mechanism, whether it be a diffuse interface or true molecular slip, the equations so far developed should describe the QCM response. However, we would emphasize that the result summarized by Eq. (30) is a first-order approximation and it may be necessary to use the earlier results prior to the expansions via Eqs. (24) and/or Eq. (27).

\section{B. Negative slip length and trapped mass}

Taking the slip length to be negative, the effect of Eq. (33) is to enhance the frequency decrease that is observed compared to a system with $b=0$. Equation (30) also shows that the existence of a slip parameter does not, to first order, alter the dissipation of the QCM compared to what would be expected for a crystal immersed in a liquid if the slip parameter vanished. These predictions are consistent with experimental results for immersion of a QCM with a small order rough surface in a wetting liquid, which give an enhanced frequency decrease, but little change in the motional resistance compared to a QCM with a smooth surface. ${ }^{13}$ These features in the experimentally observed response correspond to the type of behavior expected with a negative slip parameter $b$. In fact, Martin has previously argued on physical grounds that the effect on the response of a QCM would be primarily in the frequency response and that this can be modeled by using an additivity between the Kanazawa liquid response and a Sauerbrey term representing the trapped mass of liquid (see Ref. 13 and references therein). The requirement to be satisfied for this to occur is that the lateral scale of the surface roughness should be less than the penetration depth; otherwise, the trapped liquid may not act as a rigid mass. The result in Eqs. (31)-(33) would support the additivity argument and provide an indication of under what circumstances this argument might fail. For larger length scales of roughness, mode conversion and enhanced damping are more likely to occur and the model in Sec. II would not then be appropriate. Moreover, the results in Sec. II do not necessarily imply that roughness accounts for all experimental data that show anomalous responses. One further requirement to be able to match the application of a slip boundary condition to the response of a rough QCM to the trapped mass argument for such a QCM is to provide a physical argument for the magnitude and sign of the slip parameter $b$.

\section{Slip length and interfacial boundary}

The mathematical development for the response of the QCM uses an essentially one-dimensional model, whereas surface roughness or topographic structuring introduces a two-dimensional aspect to the problem as the thickness of crystal varies with lateral position. In this subsection we consider how the results for such a QCM surface, for small height variations compared to the crystal thickness, might be interpreted using the results of the essentially onedimensional model. Consider Fig. 3, but now imagine that the true QCM surface is rough. For simplicity, we show in Fig. 3 a dotted line giving a step-type "roughness" variation in the position of the QCM surface with equal lengths for the low and high positions (1:1 mark-space ratio). The average position of the surface is the solid horizontal line at $z=0$ and the surface features vary from $-A$ to $+A$. If we now immerse the QCM, then each corrugation of depth $2 A$ would contain trapped liquid. Within the slip boundary condition model we can imagine that this liquid is spread out across each surface feature as a mass layer of thickness $A$ 
a)

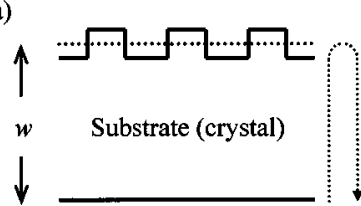

b)

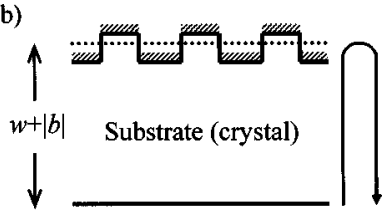

FIG. 4. Trapped mass viewed as a movement of the average center of reflection of the QCMs upper surface. (a) The unloaded QCM has a resonant frequency determined by the acoustic reflection shown the dotted arrow. (b) The trapped liquid mass in the surface features is viewed as increasing the thickness of the substrate at both the trough and crest positions of the roughness by an equal amount $b$ and so increasing the effective reflection path by $|b|$ as shown by the solid arrow.

[Fig. 4(b)]. The net effect is that the average position of the interface moves towards the bulk liquid by a distance $A$. For this particular geometry, we would argue that the slip parameter $b$ would therefore be negative and of magnitude $A$. A similar argument could be made for any other type of model surface roughness, such as a sinusoidally varying surface, and so be used to determine the slip parameter magnitude. This interpretation of a negative slip parameter means that the application of the slip boundary condition to this problem of surface roughness does not represent molecular slip, but does show that a slip boundary condition can convert the response of a QCM with a rough surface into an equivalent response for a QCM with a smooth surface. There is nothing in this particular application of the slip boundary condition that would preclude its use to also describe molecular slip, although we would expect that molecular slip would require a positive slip length parameter $b$.

\section{Acoustic reflection considerations}

The slip model matches boundary conditions at the solid-liquid interface and, with a negative $b$, merely moves the position of that interface out towards the liquid by a constant amount; this is illustrated in Figs. 4(a) and 4(b). When a QCM is in air [Fig. 4(a)] the acoustic wave in the substrate will undergo reflections from both the peaks and troughs of the QCM's corrugated upper surface, thus defining two characteristic resonant cavity lengths. Each of these cavities will define resonances of the crystal and so give two different resonant frequencies. Provided the depth of the surface features is small, adding the waves giving these two resonances will give an average resonant frequency modulated by a low-frequency variation. In effect, we could view the QCM's upper surface as having an average center of reflection so that the substrate thickness is $w$. In Fig. 4(a) the path of the acoustic reflection is shown by the dotted vertical arrow and this determines the resonant frequency. When the rough surface is completely wetted the acoustic reflectivity of the upper solid surface of the QCM is the same irrespective of whether the horizontal location ( $x$ position) corresponds to a peak or a trough in the surface corrugation/ roughness. We can imagine the trapped liquid mass being spread in a uniform film of thickness $|b|$ across the peaks and troughs of the QCM's upper surface [Fig. 4(b)]. This results in an overall and uniform shift in the average center of acoustic reflectivity towards the bulk liquid phase by an

amount $|b|$. The path of the acoustic reflection therefore increases as shown schematically by the solid vertical arrow in Fig. 4(b). An effective increase in the acoustic thickness of the substrate caused by the uniformly spread-out trapped mass would be expected to result in a larger resonant halfwavelength and so a lower resonant frequency. This particular conclusion is to some extent speculative, but the change in viewpoint to acoustic reflectivity does help identify a possible implicit assumption in the trapped mass model additivity formula [Eq. (31)].

The explicit limitation on the applicability of Eqs. (31)(33) to rough surfaces is that $b / \delta$ be small. However, it is also assumed that the model is independent of the particular point along the $x$ direction-i.e., that a damped shear wave oscillation into the liquid begins at the average position of the slip plane no matter what position along the $x$ direction is considered. For surface roughness features which are closely spaced this is likely to be true, but as they become further apart it is an assumption likely to fail. A further implicit assumption is that the liquid maintains contact with the surface features across the QCM-i.e., the surface is completely wetted; if it does not, the surface reflectivity may become a function of position along the interface. One interesting question that arises is whether vapor trapped in surface features could be accounted for simply by using Eqs. (31)-(33), but modified by reducing the amount of "trapped" liquid. ${ }^{13}$ It is not clear from the slip boundary condition model whether the additivity of "liquid" mass will still be valid if this partial penetration by liquid occurs. The slip model matches boundary conditions at the solid-liquid interface and merely moves the position of that interface out towards the liquid by a constant amount. Figures 4(a) and 4(b) are an attempt to interpret this movement in terms of acoustic reflections and lead to the identification of an important implicit assumption. If vapor trapping due to incomplete wetting occurs, acoustic reflections from the troughs of the surface features will be due to a solid-air interface while those from the peaks will be due to a solid-liquid interface. Since the acoustic reflectivity of the solid-air interface is significantly higher than that of a solid-liquid interface, this may limit the slip model assumption that all positions along the $x$ direction see an effective slip parameter of the same value; this could be a particular problem if the reflection was dominated by surface troughs which possessed a solid-vapor interface. In such circumstances, the validity of the additivity result [Eq. (31)] may be questionable and the trapped mass viewpoint may not then be applicable, in some circumstances, to hydrophobic rough surfaces. The potential complexity of this effect is one reason why we have qualified the results in Sec. II of this article to be applicable to the complete wetting case.

\section{CONCLUSION}

A slip boundary condition has been implemented via the wave equations for a QCM covered with a finite liquid layer. The response of the QCM on immersion in water assuming a slip length $b$ has been obtained and to first order in $b / \delta$, and assuming no mode conversion, it has been shown that the 
frequency response can be viewed rigorously as a liquid response with an additional component due to a "rigid liquid" mass; to first order the slip boundary condition does not change the dissipation beyond what would be expected from a no-slip boundary condition. For the "rigid liquid" mass to enhance the frequency decrease the slip length must be negative so that the slip plane is located on the liquid side of the average surface of the QCM. It has been argued that the slip boundary condition with a negative slip length could model the liquid response of a QCM with a rough surface. In this application of the slip boundary condition the results of the model are equivalent to a trapped mass model provided the liquid wets the whole of the rough surface. The possible importance of vapor trapping altering acoustic reflectivity of wetted versus nonwetted portions of the interface has been identified, although it remains unclear whether this could invalidate the trapped mass argument for hydrophobic rough surfaces.

\section{ACKNOWLEDGMENTS}

G.M. gratefully acknowledges financial support from the UK Engineering and Physical Sciences Research Council (EPSRC) under Grant No. GR/S30573/01. He would also like to acknowledge discussions with L. Theissen and R. W. Cernosek (Sandia National Laboratories), M. Thompson and J. Ellis (University of Toronto), and G. Hayward (Guelph University).

\section{APPENDIX A: FLUID VELOCITY AND IMPEDANCE FOR A VISCOELASTIC LAYER}

In this appendix we outline the key changes to Eqs. (1)(9) required to obtain the fluid velocity when the fluid is regarded as a viscoelastic layer rather than a Newtonian liquid. In the case of a viscoelastic layer, the Navier-Stokes equation for the fluid [Eq. (1)] is modified to

$$
\frac{G_{f}}{i \omega \rho_{f}} \nabla^{2} \underline{v}_{f}=i \omega \underline{v}_{f},
$$

where $G_{f}$ is the complex shear modulus and has liquid and solid limits of $i \omega \eta_{f}$ and $\mu_{f}$. Within the equation for the fluid velocity [Eq. (5)] the wave vector $k_{f}$ becomes

$$
k_{f}=\frac{\sqrt{-2 i}}{\bar{\delta}}
$$

where the complex penetration depth is defined by

$$
\bar{\delta}=\sqrt{\frac{2 G_{f}}{i \rho_{f} \omega^{2}}},
$$

which in the liquid limit becomes equal to the usual shearwave penetration depth. The boundary condition, Eq. (7), becomes

$$
\frac{G_{f}}{i \omega \rho_{f}}\left(\frac{\partial v_{f}}{\partial z}\right)_{z=d}=0 .
$$

The solution for the fluid velocity [Eq. (9)] becomes

$$
v_{f}(z)=2 A_{f} \exp \left(\frac{\sqrt{2 i} d}{\bar{\delta}}\right) \cosh \left[\frac{\sqrt{2 i}(z-d)}{\bar{\delta}}\right]
$$

In the evaluation of surface mechanical impedance the shear stress [Eq. (12)] becomes

$$
F_{f}=\frac{-G_{f}}{i \omega}\left(\frac{d v_{f}}{d z}\right)_{z=0},
$$

so that the general form for the impedance [Eq. (15)] becomes

$$
\begin{aligned}
Z_{L}= & -i \sqrt{\frac{i \rho_{f} \eta_{f}}{\omega}}\left(\frac{\bar{\delta}}{\delta}\right)\left(\frac{A_{f}}{A_{s}}\right) \exp \left(i k_{s} w+\frac{\sqrt{2 i} d}{\bar{\delta}}\right) \\
& \times\left[\frac{\sinh \left(\frac{\sqrt{2 i} d}{\bar{\delta}}\right)}{\cos \left(k_{s} w\right)}\right] .
\end{aligned}
$$

The key differences in Eq. (A7) compared to Eq. (15) are the replacement of the penetration depth by the complex penetration depth in the $\exp (\cdots)$ and $\sinh (\cdots)$ factors and the inclusion of a factor which is the ratio of complex penetration depth to penetration depth. Equation (A7) is a general form in the sense that it has not yet had a boundary condition imposed at the substrate-layer interface.

\section{APPENDIX B: SLIP LENGTH PARAMETER AND A VISCOELASTIC LAYER}

In this appendix the effect of the slip boundary condition on a viscoelastic layer is detailed. In the no-slip boundary condition case the relation between the $A_{f}$ and $A_{s}$ coefficients [Eq. (16)] becomes

$$
\begin{aligned}
A_{f}^{n o \text { slip }}= & i \omega \exp \left(-i k_{s} w+\frac{\sqrt{2 i} d}{\bar{\delta}}\right) \\
& \times\left[\frac{\cos \left(k_{s} w\right)}{\cosh \left[\frac{\sqrt{2 i} d}{\bar{\delta}}\right]}\right] A_{s}^{\text {no slip }},
\end{aligned}
$$

which gives the impedance

$$
Z_{L}^{n o \quad s l i p}=\sqrt{i \omega \rho_{f} \eta_{f}}\left(\frac{\bar{\delta}}{\delta}\right) \tanh \left[\frac{\sqrt{2 i} d}{\bar{\delta}}\right] .
$$

The equivalent results derived by imposing the Hayward-Ellis ${ }^{18}$ slip boundary condition [Eq. (19)] are

$$
\begin{aligned}
A_{f}^{\text {slip }}= & i \omega \exp \left(-i k_{s} w-\frac{\sqrt{2 i} d}{\bar{\delta}}\right) \\
& \times\left[\frac{\cos \left(k_{s} w\right)}{\cosh \left[\frac{\sqrt{2 i}(d+b)}{\bar{\delta}}\right]}\right] A_{s}^{\text {slip }}
\end{aligned}
$$




$$
Z_{L}^{\text {slip }}=\frac{\sqrt{i \omega \rho_{f} \eta_{f}}\left(\frac{\bar{\delta}}{\delta}\right) \sinh \left(\frac{\sqrt{2 i} d}{\bar{\delta}}\right)}{\left(\frac{\cosh \sqrt{2 i}(d+b)}{\bar{\delta}}\right)} .
$$

Expanding the denominator we obtain

$$
Z_{L}^{\text {slip }} \approx=\frac{Z^{\text {no slip }}}{1+s Z^{\text {no slip }},}
$$

where $Z_{L}^{\text {slip }}$ and $Z_{L}^{\text {no slp }}$ are defined by Eqs. (B2) and (B4) and the $s$ parameter for the viscoelastic layer is given by $s$ $=i \omega b / G_{f}$ :

$$
s=\frac{i \omega b}{G_{f}} .
$$

We note that the McHale et al. ${ }^{17}$ and Ellis-Hayward ${ }^{18}$ slip boundary conditions can be related from the Taylor expansion, Eq. (20), and the shear stress, Eq. (A6), and that doing so gives Eq. (B6).

\section{APPENDIX C: SLIP PARAMETER AND THIN RIGID MASS LIMIT}

In the case of a thin "rigid" mass layer we find, from Appendix B,

$$
Z_{L}^{\text {no slip }} \approx i \omega \rho_{f} d,
$$

which is the Sauerbrey mass response, and expanding the denominator in Eq. (B5) gives

$$
Z_{L}^{s l i p} \approx i \omega \rho_{f} d\left[1-\frac{\omega^{2} b \rho_{f} d}{\mu_{f}}\right],
$$

where $\mu_{f}$ is the solid limit of the shear modulus $G_{f}$. Thus, the usual Sauerbrey response is modified by an additional term. To gain insight into the additional term in Eq. (C2), we can rewrite Eq. (C2) as

$$
Z_{L}^{s l i p} \approx i \omega \rho_{f} d\left[1-\left(\frac{2 \pi b}{\lambda_{f}}\right)\left(\frac{2 \pi d}{\lambda_{f}}\right)\right],
$$

where the wavelength in the fluid has been defined as

$$
\lambda_{f}=\frac{2 \pi}{\omega} \sqrt{\frac{\mu_{f}}{\rho_{f}}} .
$$

For small slip length and layer thickness compared to the wavelength [Eq. (C4)], Eq. (C3) shows that the correction to the Sauerbrey equation is small.

${ }^{1}$ R. Schumacher, G. Borges, and K. K. Kanazawa, Surf. Sci. 163, L621 (1985).

${ }^{2}$ R. Schumacher, J. G. Gordon, and O. Melroy, J. Electroanal. Chem. Interfacial Electrochem. 216, 127 (1987).

${ }^{3}$ R. Schumacher, J. Electroanal. Chem. Interfacial Electrochem. 219, 311 (1987).

${ }^{4}$ R. Schumacher, Angew. Chem., Int. Ed. Engl. 29, 329 (1999).

${ }^{5}$ M. Thompson, A. L. Kipling, W. C. Duncan-Hewitt, L. V. Rajakovic, and B. A. Cavic-Vlasak, Analyst (Cambridge, U.K.) 116, 881 (1991).

${ }^{6}$ R. Beck, U. Pitterman, and K. G. Weil, J. Electrochem. Soc. 139, 453 (1992).

${ }^{7}$ D. M. Soares, Meas. Sci. Technol. 4, 549 (1993).

${ }^{8}$ S. J. Martin, G. C. Frye, and A. J. Ricco, Anal. Chem. 65, 2910 (1993).

${ }^{9}$ M. Yang and M. Thompson, Langmuir 9, 1990 (1993).

${ }^{10}$ S. J. Martin, G. C. Frye, A. J. Rocci, and S. D. Senturia, Anal. Chem. 65, 2910 (1993)

${ }^{11}$ M. Yang, M. Thompson, and W. C. Duncan-Hewitt, Langmuir 9, 802 (1993).

${ }^{12}$ L. Daikhin and M. Urbackh, Faraday Discuss. 107, 27 (1997).

${ }^{13}$ S. J. Martin, Faraday Discuss. 107, 463 (1997).

${ }^{14}$ K. K. Kanazawa and J. G. Gordon, Anal. Chem. 57, 1770 (1985).

${ }^{15}$ G. Sauerbrey, Z. Phys. 155, 206 (1959).

${ }^{16}$ M. Rodahl and B. Kasemo, Sens. Actuators A 54, 448 (1996).

${ }^{17}$ G. McHale, R. Lucklum, M. I. Newton, and J. A. Cowen, J. Appl. Phys. 88, 7304 (2000).

${ }^{18}$ J. S. Ellis and G. L. Hayward, J. Appl. Phys. (accepted for publication).

${ }^{19}$ B. A. Cavic, F. L. Chu, L. M. Furtado, S. Ghafouri, G. L. Harward, D. P. Mack, M. E. McGovern, H. Su, and M. Thompson, Faraday Discuss. 107, 159 (1997).

${ }^{20}$ W. C. Duncan-Hewitt and M. Thompson, Anal. Chem. 64, 94 (1992).

${ }^{21}$ S. J. Martin, V. E. Granstaff, and G. C. Frye, Anal. Chem. 63, 2272 (1991).

${ }^{22}$ G. McHale, M. I. Newton, and F. Martin, J. Appl. Phys. 91, 9701 (2002).

${ }^{23}$ C. Behling, Ph.D. thesis, University of Magdeburg, 1999. 